\title{
DRIVER CONTROL ACTIONS IN HIGH-SPEED CIRCULAR DRIVING
}

\author{
Diomidis Katzourakis ${ }^{1}$, Efstathios Velenis ${ }^{2}$, \& Riender Happee ${ }^{1}$ \\ ${ }^{1}$ Biomechanical Engineering Research Group, Mechanical, Maritime, and Materials \\ Engineering, Delft University of Technology, Delft, The Netherlands \\ ${ }^{2}$ School of Engineering and Design, Brunel University, Uxbridge, UK \\ Email: d.katzourakis@tudelft.nl
}

\begin{abstract}
Summary: In this pilot study we investigate driver control actions during high speed cornering with a rear wheel drive vehicle. Six drivers were instructed to perform the fastest maneuvers possible around a marked circle, while trying to retain control of the vehicle and constant turning radius. The data reveal that stabilization of the vehicle is achieved with a combination of steering and throttle regulation. The results show that the drivers used steering control to compensate for disturbances in yaw rate and sideslip angle. Vehicle accustomed drivers had the most consistent performance resulting in reduced variance of task metrics and control inputs.
\end{abstract}

\section{INTRODUCTION}

Driving control analysis studies were initiated as early as in the 1930's (Gibson \& Crooks, 1938). It was soon realized that the driving task can be divided into a leading and a compensation action and that drivers primarily apply steering in an anticipatory feedforward manner to an estimated future path; in addition, drivers employ a closed-loop adaptive-control strategy to compensate for deviations of the vehicle from the demanded trajectory (McRuer \& Krendel, 1974). The dominant approach in the design of human-like driver controllers is to decouple the anticipatory and compensatory actions (e.g. Edelmann et al., 2007); however, the full understanding of human driving in terms of compensation to steering disturbances (e.g. Katzourakis et al., 2010) remains an open issue. The majority of driver-car interaction studies dealing with the driver's compensatory behaviour are performed in a simulation environment (e.g. Odhams \& Cole, 2010) since real in-field extreme driving tests can be difficult to interpret (e.g. Breuer, 1998). Expert rally driving techniques and their corresponding mathematical analysis, which involves operation of the vehicle outside the stable operation envelope has recently started to receive attention (Velenis et al., 2007a, b). The former invited the introduction of vehicle stabilization controllers employing solely driver inputs (Velenis et al., 2010).

Challenged by the human's compensatory behaviour while driving beyond the vehicle's stable envelope, we commenced a pilot study to investigate the relationship between driver's sensory inputs and compensatory control-actions. The sensory inputs can be visual, kinesthetic (steering torque) or vestibular (lateral acceleration, yaw rate and slip angle) feedback. Six drivers with varying driving skill level were instructed to execute high-speed circular maneuvers on a loose surface (dirt), aiming at maintaining approximately a constant sideslip angle and distance from the center of the tire-marked circular path (with $7.5 \mathrm{~m}$ radius). By analyzing the driver control actions and the vehicle response, we studied the cross-correlation of the sensory inputs and the corresponding control actions (steering, throttle). 


\section{METHODS}

The tests took place at the facilities of the Bill Gwynne Rally School in Brackley, UK, using a rally-race prepared rear-wheel-drive (RWD) 1980 Ford Escort Mk1 with a 1.6 liter engine producing approximately $110 \mathrm{bhp}$ (Figure 1). A VBOXIISL data-logger from Racelogic was used to measure the vehicle's absolute position, true heading, velocity and sideslip angle $\beta$. A low cost Inertia Measurement Unit (IMU) with 5 degrees-of-freedom IDG500/ADXL335 was placed near the estimated location of the vehicle's centre-of-gravity (CG) to measure 3-axis body accelerations and 2-axis body angular rates. Externally fitted optical encoders (speed sensors) were used to measure the rotational speed of individual wheels. The steering angle/torque signals were measured using an 'extension hub' mounted between the steering wheel hub and the steering wheel. Strain gauges on the 'extension hub' enabled steering torque reading and a string potentiometer wrapped around the 'extension hub' measured the steering wheel angle. Throttle position was measured through a potentiometer. The vehicle was fitted with two brake pressure sensors allowing us to distinguish between application of foot brake and handbrake. A National Instruments USB-6211 USB M Series data-acquisition was used to capture the analog signals and an 8-bit AVR ATMega32 microcontroller was used for interfacing the optical encoders of the wheel speed sensors. The data logging was performed at $100 \mathrm{~Hz}$ on a Toshiba NB200 notebook. In-house developed software, based exclusively on open-source solutions, handled the logging and synchronization process (Katzourakis et al., 2011a). The vehicle instrumentation is shown in Figure 1.
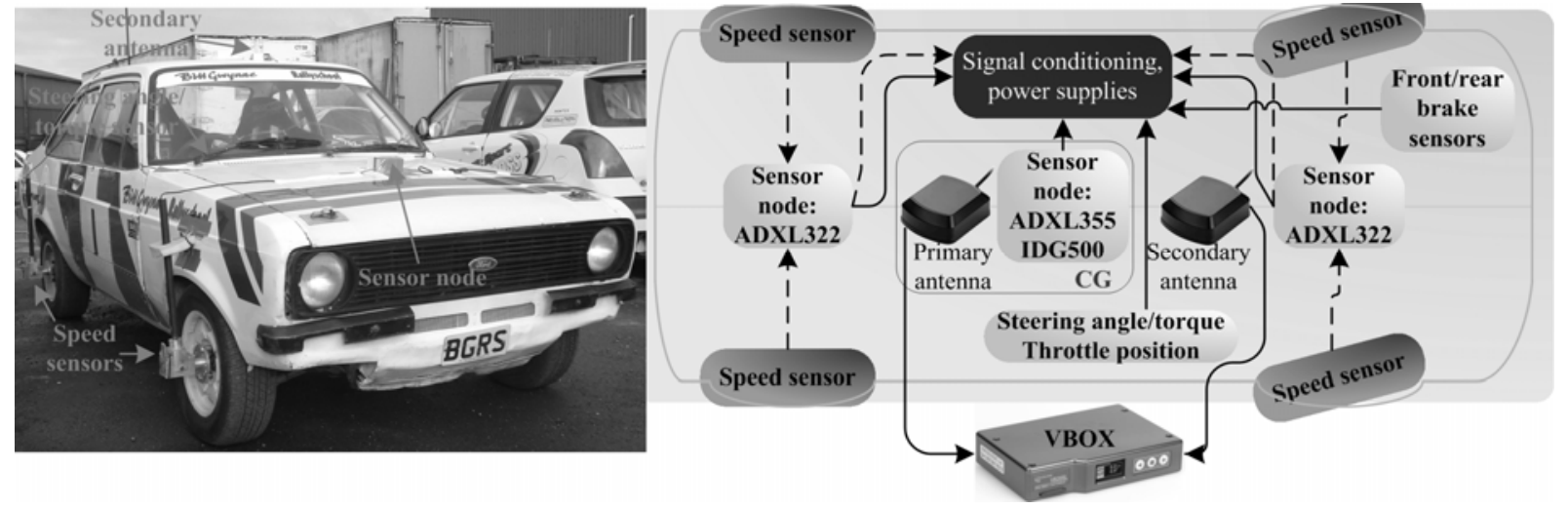

Figure 1. Vehicle instrumentation for data recording

Three drivers (D1, D2, D3) with extensive racing experience (expert drivers) and three with no racing experience (D4, D5, D6) (normal drivers) were employed for testing. Each driver was asked to perform three sessions of at least two clockwise circular runs. High speed cornering at high sideslip angles involves operation of the vehicle in an unstable regime (Velenis et al., 2010) and hence is a challenging control task. The drivers were instructed to use only throttle and steering to regulate the vehicle, so as to make a simplified one-to-one relationship (Table 2) between driver inputs and vehicle's response in the absence of tire force data.

Mean and standard deviation of several task related metrics were calculated for each test. The mean values describe the steady-state condition achieved. The standard deviations describe deviating vehicle kinematics emerging from physical disturbances, such as variations of tire grip 
which are compensated by the human controller. As described below, we relate the measured control actions to the kinematic deviations. The vehicle states are the velocity $V$, the sideslip angle $\beta$ and the yaw rate $\dot{\psi}$ (Figure 2; left); Table 1 summarizes the vehicle variables.

Throughout the paper we assume that the vehicle operates near a steady-state cornering condition. Under this assumption, the vehicle sketches a circular trajectory with radius $R$ tangent to the velocity vector $V$ Figure 2; left). The radius $R$ of the circle can be calculated using (1). Counter-clockwise rotation corresponds to a positive yaw rate (Figure 2; right) and therefore positive $R$. Referring to Figure 2 we define $D$ as the distance of the car's $\mathrm{CG}([X, Y])$ to the center $C_{M}$ of the marked path; thus $D$ is always greater than or equal to 0 .

$$
\begin{gathered}
R=V / \dot{\psi} \\
R \text { Visual }=R-\operatorname{sign}(R) \cdot D
\end{gathered}
$$

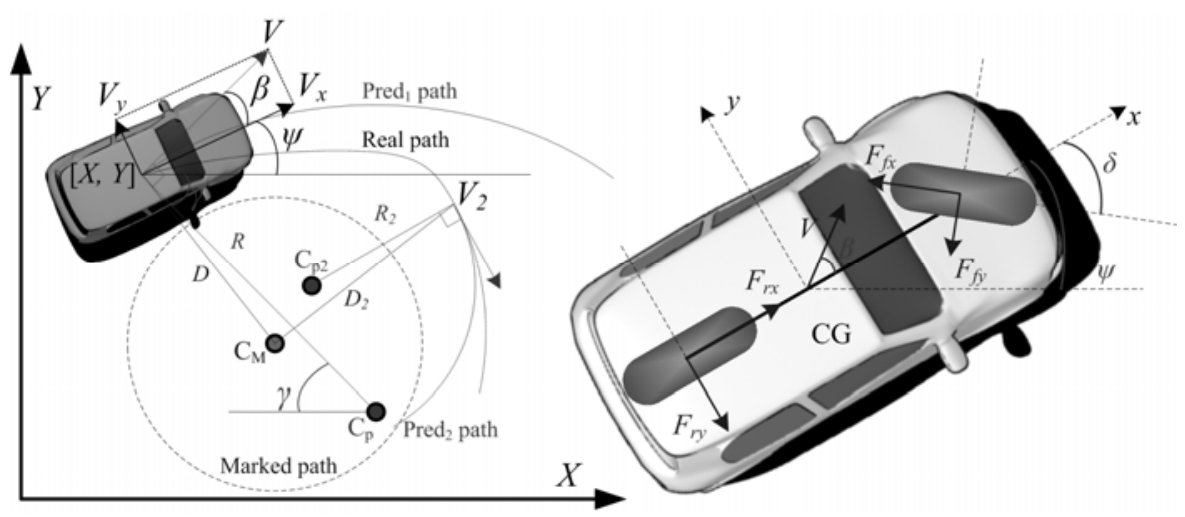

Figure 2. Vehicle's predicted path (left) and cornering model with forces (right); $R<0$

Table 1. Vehicle variables nomenclature

\begin{tabular}{cl}
\hline$V, \delta$ & Velocity, steering angle \\
$F_{f y}, F_{r y}$ & Lateral forces: front, rear axle \\
$F_{f x}, F_{r x}$ & Tractive forces: front, rear axle \\
$X, Y$ & Global frame coordinates: $X, Y$ \\
$x, y, \psi$ & Vehicle frame coordinates: $x, y$, yaw angle \\
$\theta_{s w}, \theta_{t h}$ & Steering wheel angle, throttle angle \\
acc $_{y}, \beta, \dot{\psi}$ & Lateral acceleration, sideslip angle, yaw rate \\
\hline
\end{tabular}

We define the relationship between the driver's sensory inputs and control actions as "acting" so as to achieve a task or "counteracting" so as to compensate an unexpected disturbance. As sensory inputs we consider the $1^{\text {st }}$ order derivatives of $R$ Visual (2), yaw rate, lateral acceleration $a c c_{y}$ and sideslip angle $\beta$. As control actions we consider the $1^{\text {st }}$ order derivatives of the steering $\theta_{s w}$ and $\theta_{t h}$ throttle angle. The differentiated signals are low pass filtered at $2.5 \mathrm{~Hz}$ with a zerophase $3^{\text {rd }}$ order Butterworth filter. The relationships between sensory inputs and control actions are defined in Table 2. An example is shown in Figure 3, showing instances from the relationship 3 of Table 2.. $\Delta \mathrm{t}$ (Figure 3 ) is the lead-lag time difference where the sensory input and the control signal have their maximum overlay (coherence); always with the sensory input being the 
reference. When a relationship is "acting", the control should lead the sensory input $(\Delta t<0)$. In a "counteracting" relationship the sensory input should lead the control $(\Delta t \geq 0)$; otherwise the sample is discarded. The relationships are denominated in Table 2 as "acting" or "counteracting" according to which cross-correlation combination (positive $(+)$ or negative $(-)$ control) between the sensory input and control action gives the greatest coherence value (coherence $=$ maximum value of the cross-correlation sequence).

When $\Delta t \geq 0$, we shall call it lag time. The samples in Figure 3 shown as discarded did not support the lead-lag time criteria of the relationship. The displayed control signal is shifted by the $\Delta \mathrm{t}$ time with the respect to the sensory input signal, at the time where both signals have their maximum coherence. The cross-correlation of the sensory input and the control action is being calculated at the switching points where the sensory input crosses zero (derivative zero $\rightarrow$ change of direction in the signal) for $T_{\text {ahead }}$ time ahead in the future. The sensory input signal within $T_{\text {ahead }}$ range should have a maximum value above the $85 \%$ of the values of the whole length of the signal; otherwise we assume that the sensory input cannot excite adequately a compensatory response from the driver and the sample is discarded.

Table 2. Relationships between driver's sensory inputs and control actions

\begin{tabular}{|c|c|c|c|c|c|c|}
\hline \multirow{3}{*}{ Sensory inputs } & \multicolumn{6}{|c|}{ Control actions } \\
\hline & \multicolumn{3}{|c|}{$\operatorname{StEERING:} \dot{\theta}_{s w}$} & \multicolumn{3}{|c|}{$\begin{array}{c}\text { Throttle angle: } \dot{\theta}_{t h} \\
(R \geq 0 \text { case; inverse }+ \text { and }- \text { for } \mathrm{R}<0)\end{array}$} \\
\hline & $(+)$ & & $(-)$ & $(+)$ & & $(-)$ \\
\hline$d R$ Visual/dt $(+)$ & counteracting & 1 & acting & counteracting & 2 & acting \\
\hline$\ddot{\psi}(+)$ & acting & 3 & counteracting & acting & 4 & counteracting \\
\hline$d a c c_{y} / d t(+)$ & acting & 5 & counteracting & acting & 6 & counteracting \\
\hline$\dot{\beta}(+)$ & counteracting & 7 & acting & counteracting & 8 & acting \\
\hline
\end{tabular}
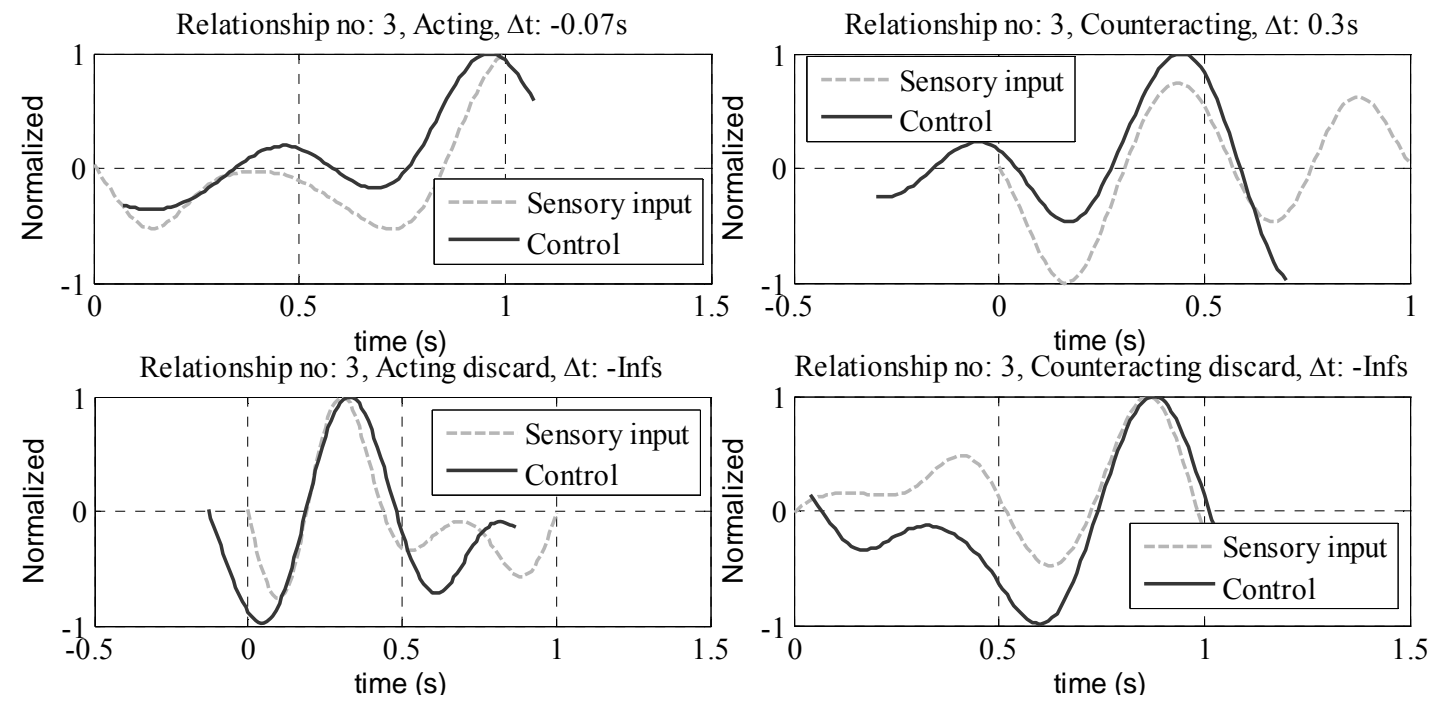

Figure 3. Acting/counteracting relationship $3 ; 1^{\text {st }}$ time derivatives of yaw rate (sensory input) and steering wheel angle (control); signals are normalized to lie within $[-1,1]$ in $T_{\text {ahead }}$ range before being cross-correlated 
Consider, for example, the visual feedback RVisual sensory input defined in (2). Assuming a clockwise turn, the radius $R$ will be negative according to (1). Now if $R$ Visual $<0(-R>D \rightarrow$ $|R|>D$ ) we expect that if the driver does not correct for his/her future path, he/she will drive away from the Marked path (Figure 2 left; case $V$ ). He/she should therefore control towards reducing the magnitude of the radius $R$. The driver may reduce the magnitude of $\mathrm{R}$ by increasing the applied steering command towards the direction of the corner (Gillespie, 1992), which corresponds to relationship 1 in Table 2. The inverse will happen if $|R|<D$. The driver should then increase the turning radius (Figure 2 left; case $V_{2}$ ); otherwise he/she will cross the marked path (or might perform a trajectory which does not even encircle the marked path). The relationships 4, 8 between the application of throttle and the induced oversteer (increase in magnitude of yaw rate and sideslip angle) can be explained if we recall the tire force mechanism under combined acceleration and cornering as discussed in Velenis et al., (2010). Essentially, the application of throttle and resulting increase in tire slip ratio results in a decrease of the stabilizing yaw moment of the rear tires, which is experienced as an increase of the vehicle yaw rate and sideslip angle. We observe that the $\dot{\theta}_{t h}$ control relationships in Table 2 depend on the sign of the radius, which is due to the fact that the $\theta_{t h}$ is an unsigned variable (in contrast to the $\left.\theta_{s w}\right)$, normalized in the $0-1$ range, representing off-throttle and full-throttle correspondingly. Therefore, its impact on the vehicle response differs according to the vehicles' states.

\section{RESULTS}

Figure 4 shows mean and standard variation $(\sigma)$ of several task related metrics (velocity, slip angle etc.) for the circular test drives. It additionally shows results of the coherence analysis by presenting the calculated lag time for all the relationships of Table 2 (using only instances determined to be counteracting; $\Delta \mathrm{t} \geq 0$ in Figure 3) for all six drivers for their $1^{\text {st }}$ and $3^{\text {rd }}$ test run using $T_{\text {ahead }}=1 \mathrm{~s}$. The drivers D1, D2, and D3 have race driving experience with the driver D1 having the greatest race distinction. Drivers D4, D5 and D6 have no race record. Drivers D1, D2 and D5 were accustomed with the test vehicle. All drivers besides driver D3 achieved their maximum mean speed in their $1^{\text {st }}$ run, which was reduced in their $2^{\text {nd }}$ run and increased again in the final $3^{\text {rd }}$ run. The mean velocity $V$ ranged from 6.2 to $7.5 \mathrm{~m} / \mathrm{s}$ and the magnitude of the mean lateral acceleration that all drivers achieved was approximately $0.5 \mathrm{~g}$, with approximately $0.15 \mathrm{~g}$ $\sigma$. Driver's D3 and D6, lost completely the control of the vehicle during their $1^{\text {st }}$ run and had to start accelerating again from standstill. D3 continued the $1^{\text {st }}$ run counterclockwise after losing control. Only the second, successful parts of these tests have been analyzed. The performance of D1, D2 (the drivers which achieved best circular trajectories) increased with the number of runs. This can be seen by the small (compared to the rest of the test group) $\sigma$ on the vehicle's states (velocity, yaw rate and slip angle) and steering angle. A small $\sigma$ on the steering shows small corrections on the steering wheel to retain control of the vehicle; a characteristic found in expert drivers. A high $\sigma$ on the throttle angle means that the driver is controlling the vehicle using the rear wheel slip; characteristic again of an expert driver (the throttle response is direct for the race-specifications test vehicle used). Driver D6 was the least trained driver; still though, though conservative driving ( small $V$ ) he managed a reduced variance of the task metrics in his $3^{\text {rd }}$ run. Drivers D1, D2 and D5 have the steadiest behaviour in terms of their achieved trajectories and relationship results, the most consistent of the test group. Lack of experience with the test vehicle induced the distinguished race driver D3 to perform worse than expected. In order for a relationship of Table 2 to give a reasonable lag time we should anticipate a $\sigma$ which is at least 
half that of the corresponding mean; also the mean should remain within similar levels over the same test driver for all the 3 test runs. The relationships 3 and 7, describing the driver's steering reaction on a yaw rate and slip angle changes correspondingly are the most consistent. Relationship 3 has approximately $\Delta \mathrm{t}=0.35 \mathrm{~s}$ lag time with $\sigma$ of 0.12 . Relationship 7 has a smaller lag time, but at the same time, a smaller $\sigma$. Relationship 5, describing the driver's steering angle reaction on lateral acceleration change, although it gives reasonable results has great variability. Relationship 1 describing the control reactions to visual sensory inputs has small coherence. This result was anticipated since the drivers during testing seem to minimally rely on their visual feedback to compensate for disturbances in the vehicle's response (Katzourakis et. al, 2011a).

\begin{tabular}{|c|c|c|c|c|c|c|c|c|c|c|c|c|c|}
\hline & Driver & \multicolumn{4}{|c|}{ D1 } & \multicolumn{4}{|c|}{ D2 } & \multicolumn{4}{|c|}{ D3 } \\
\hline & Run & \multicolumn{2}{|c|}{$1 \mathrm{st}$} & \multicolumn{2}{|c|}{$3 \mathrm{rd}$} & \multicolumn{2}{|c|}{$1 \mathrm{st}$} & \multicolumn{2}{|c|}{$3 \mathrm{rd}$} & \multicolumn{2}{|c|}{$1 \mathrm{st}$} & \multicolumn{2}{|c|}{ 3rd } \\
\hline & & $\mu$ & $\sigma$ & $\mu$ & $\sigma$ & $\mu$ & $\sigma$ & $\mu$ & $\sigma$ & $\mu$ & $\sigma$ & $\mu$ & $\sigma$ \\
\hline & $V_{(\mathrm{m} / \mathrm{s})}$ & 6,93 & 0,82 & 6,67 & 0,88 & 7,51 & 0,66 & 6,69 & 0,78 & 6,45 & 1,89 & 7,07 & 0,74 \\
\hline & $d \psi / d t\left(^{o}\right)$ & $-33,04$ & 21,70 & $-38,44$ & 7,34 & $-32,90$ & 16,09 & $-39,98$ & 11,21 & 27,88 & 9,23 & $-37,18$ & 21,81 \\
\hline & $\beta\left(^{o}\right)$ & 14,84 & 15,72 & 2,36 & 3,53 & 12,14 & 11,24 & 4,39 & 6,05 & $-1,75$ & 2,50 & 12,10 & 14,39 \\
\hline & $a c c_{y}(g)$ & $-0,46$ & 0,13 & $-0,54$ & 0,13 & $-0,55$ & 0,16 & $-0,51$ & 0,13 & 0,39 & 0,19 & $-0,49$ & 0,15 \\
\hline & RVisual & $-1,64$ & 28,54 & $-0,38$ & 2,15 & $-5,62$ & 23,74 & 1,09 & 15,37 & 0,37 & 3,53 & $-2,58$ & 16,17 \\
\hline & $\theta_{s w}\left({ }^{0}\right)$ & $-56,44$ & 256,01 & $-231,80$ & 52,70 & $-90,82$ & 153,78 & $-215,71$ & 81,87 & 271,86 & 121,03 & $-68,05$ & 209,78 \\
\hline & $\theta_{t h}($ norm $)$ & 0,75 & 0,22 & 0,82 & 0,13 & 0,77 & 0,25 & 0,71 & 0,14 & 0,61 & 0,18 & 0,81 & 0,14 \\
\hline \multirow{18}{*}{ 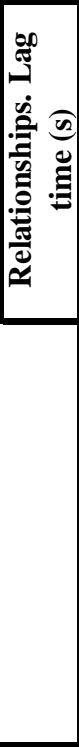 } & 1 & 0,18 & 0,08 & 0,37 & 0,10 & 0,26 & 0,25 & 0,21 & 0,21 & 0,40 & 0,27 & 0,29 & 0,19 \\
\hline & 2 & 0,70 & 0,09 & 0,32 & 0,21 & 0,67 & 0,15 & 0,28 & 0,36 & 0,15 & 0,26 & 0,28 & 0,21 \\
\hline & 3 & 0,32 & 0,12 & 0,38 & 0,13 & 0,44 & 0,15 & 0,34 & 0,15 & 0,36 & 0,11 & 0,22 & 0,09 \\
\hline & 4 & 0,29 & 0,19 & 0,24 & 0,17 & 0,32 & 0,21 & 0,14 & 0,15 & 0,27 & 0,27 & 0,22 & 0,22 \\
\hline & 5 & 0,27 & 0,15 & 0,29 & 0,21 & 0,27 & 0,21 & 0,23 & 0,16 & 0,19 & 0,13 & 0,26 & 0,13 \\
\hline & 6 & 0,43 & 0,23 & 0,28 & 0,22 & 0,37 & 0,24 & 0,45 & 0,28 & 0,49 & 0,24 & 0,27 & 0,21 \\
\hline & 7 & 0,18 & 0,05 & 0,28 & 0,16 & 0,10 & 0,08 & 0,29 & 0,09 & 0,24 & 0,19 & 0,12 & 0,04 \\
\hline & 8 & 0,20 & 0,18 & 0,26 & 0,19 & $\mathrm{NaN}$ & $\mathrm{NaN}$ & 0,29 & 0,24 & 0,37 & 0,22 & 0,12 & 0,17 \\
\hline & Driver & \multicolumn{4}{|c|}{$\mathrm{D} 4$} & \multicolumn{4}{|c|}{ D5 } & \multicolumn{4}{|c|}{ D6 } \\
\hline & Run & \multicolumn{2}{|c|}{ 1st } & \multicolumn{2}{|c|}{3 rd } & \multicolumn{2}{|c|}{$1 \mathrm{st}$} & \multicolumn{2}{|c|}{ 3rd } & \multicolumn{2}{|c|}{$1 \mathrm{st}$} & \multicolumn{2}{|c|}{ 3rd } \\
\hline & & $\mu$ & $\sigma$ & $\mu$ & $\sigma$ & $\mu$ & $\sigma$ & $\mu$ & $\sigma$ & $\mu$ & $\sigma$ & $\mu$ & $\sigma$ \\
\hline & $V_{(\mathrm{m} / \mathrm{s})}$ & 7,15 & 0,71 & 6,82 & 0,98 & 6,70 & 0,94 & 6,79 & 0,85 & 6,30 & 0,76 & 6,20 & 0,56 \\
\hline & $d \psi / d t\left(^{o}\right)$ & $-36,70$ & 17,44 & $-33,68$ & 24,49 & $-37,66$ & 17,28 & $-40,42$ & 12,00 & $-34,11$ & 19,52 & $-35,29$ & 11,98 \\
\hline & $\beta\left(^{\circ}\right)$ & 9,17 & 10,17 & 10,26 & 14,41 & 6,67 & 7,40 & 9,39 & 8,50 & 5,58 & 9,14 & 0,75 & 2,34 \\
\hline & $a c c_{y}(g)$ & $-0,51$ & 0,13 & $-0,45$ & 0,18 & $-0,51$ & 0,19 & $-0,50$ & 0,13 & $-0,44$ & 0,16 & $-0,43$ & 0,15 \\
\hline & RVisual & $-2,82$ & 19,52 & $-0,07$ & 29,06 & 0,72 & 18,22 & $-0,66$ & 19,06 & $-0,16$ & 12,88 & $-1,97$ & 8,91 \\
\hline & $\theta_{s w}\left({ }^{0}\right)$ & $-168,32$ & 192,14 & $-117,90$ & 214,48 & $-207,32$ & 143,82 & $-150,33$ & 142,22 & $-207,38$ & 165,30 & $-237,20$ & 84,03 \\
\hline & $\theta_{\text {th }}($ norm $)$ & 0,77 & 0,15 & 0,69 & 0,18 & 0,78 & 0,15 & 0,77 & 0,17 & 0,70 & 0,24 & 0,66 & 0,14 \\
\hline \multirow{8}{*}{ 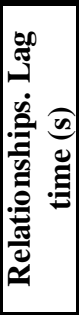 } & 1 & 0,34 & 0,17 & 0,38 & 0,23 & 0,32 & 0,25 & 0,32 & 0,22 & 0,33 & 0,29 & 0,42 & 0,00 \\
\hline & 2 & 0,47 & 0,15 & 0,19 & 0,17 & 0,07 & 0,03 & 0,17 & 0,12 & 0,34 & 0,31 & 0,13 & 0,08 \\
\hline & 3 & 0,43 & 0,17 & 0,35 & 0,07 & 0,38 & 0,10 & 0,33 & 0,12 & $\mathrm{NaN}$ & $\mathrm{NaN}$ & 0,33 & 0,08 \\
\hline & 4 & 0,40 & 0,29 & 0,34 & 0,09 & 0,29 & 0,23 & 0,28 & 0,21 & 0,11 & 0,00 & 0,24 & 0,14 \\
\hline & 5 & 0,27 & 0,20 & 0,41 & 0,10 & 0,28 & 0,20 & 0,33 & 0,22 & 0,73 & 0,00 & 0,40 & 0,11 \\
\hline & 6 & 0,31 & 0,25 & 0,31 & 0,20 & 0,30 & 0,13 & 0,49 & 0,20 & 0,17 & 0,20 & 0,41 & 0,26 \\
\hline & 7 & 0,35 & 0,03 & 0,10 & 0,11 & 0,22 & 0,11 & 0,20 & 0,14 & 0,12 & 0,06 & 0,30 & 0,09 \\
\hline & 8 & 0,44 & 0,04 & 0,01 & 0,00 & 0,19 & 0,23 & 0,37 & 0,25 & 0,40 & 0,02 & 0,24 & 0,18 \\
\hline
\end{tabular}

Figure 4. Mean $\mu$ and standard deviation $\sigma$ for vehicle's signals, control actions and lag times of the relationships of

Table 2; $(\mathrm{NaN}=$ no sample passed the selection process $)$ 


\section{DISCUSSION}

In this pilot-study we discussed the human's compensatory behaviour and driving outside the vehicle's stable envelope where considerable control effort is required to retain stability. Six different test drivers executed high-speed circular maneuvers on a loose surface, instructed to maintain constant sideslip angle and distance from the center of the tire-marked circular path. By employing a method which relates the driver's sensory inputs with control actions we can get a notion of their coupled relationship and their lead-lag time difference. From the results, we can see that a driver will likely counteract through steering to an exciting enough change (sensory input signal should contain a maximum value, above the $85 \%$ of the values of the whole length of the signal) of the yaw rate and the slip angle, with a time delay of approximately 0.4 and $0.2 \mathrm{~s}$ correspondingly. Drivers accustomed with the test vehicle exhibit smaller standard deviation $(\sigma)$ in their steering inputs and the realized vehicle's states and higher $\sigma$ in the throttle control. They also display more consistent lag times for the relationships of Table 2 with small variation in the mean (per run) and low standard deviation in total. The metrics could therefore be used to asses driving skills. The employed method is sensitive because of the complex nature of the driver-car system and despite the fact that the selection process rejects multiple outliers; the results can be still debatable. This pilot study does not allow drawing definite conclusions about the relationships. The application of classical system-identification techniques (Katzourakis et al., 2011b), which would perturb the vehicle's response by applying a known disturbance would give more rigorous conclusions. Still, the suggested method can potentially give fruitful results by expanding the test group. Definitely though, being a race driver assures high performance, but being accustomed with the test vehicle seems also to be of great importance.

\section{ACKNOWLEDGMENT}

The work of Diomidis Katzourakis is supported by the Automotive Development Center of SKF as part of the project Mobility Intelligence using Loadbased Lateral Stability (MILLS).

Efstathios Velenis is supported by a Marie Curie International Reintegration Grant within the 7th European Community Framework Programme.

\section{REFERENCES}

Breuer, J.J. (1998). Analysis of Driver-Vehicle-Interaction in An Evasive Manoueuvre - Results of Moose Test Studies. Proc. of the 16th ESV Conference, Paper No: 98-S2-W-35.

Edelmann, J., Plöchl, M., Reinalter, W. \& Tieber, W. (2007). A passenger car driver model for higher lateral accelerations. Vehicle System Dynamics, 45(12), 1117-1129.

Gibson, J.J. \& Crooks, L.E. (1938). A theoretical field-analysis of automobile-driving. American Journal of Psychology, 51, 453-471.

Gillespie, T.D. (1992). Fundamental of Vehicle Dynamics. SAE, 1992.

Katzourakis, D., Droogendijk, C., Abbink, D., Happee, R. \& Holweg, E. (2010). Driver Model with Visual and Neuromuscular Feedback for Objective Assessment of Automotive Steering Systems. Proc. of the 10th Int. Symp. on Advanced Vehicle Control, AVEC10, 381-386. 
Katzourakis, D., Velenis, E., Abbink, A., Happee, R. \& Holweg, E. (2011a). Race Car Instrumentation for Racing Behaviour Studies. IEEE Trans. on Instrumentation and Measurement, to appear (2011).

Katzourakis, D., Abbink, D.A., Happee, R. \& Holweg, E. (2011b). Steering Force Feedback for Human-Machine-Interface Automotive Experiments. IEEE Trans. on Instrumentation and Measurement, 60 (1), 32-43.

McRuer, D.T. \& Krendel, E. (1974). Mathematical Models of Human Pilot Behavior. AGARD AG 188, STI-P-146. Available: http://www.systemstech.com/.

Odhams, A.M.C. \& Cole, D.J. (2010). Identification of a driver's preview steering control behaviour using data from a driving simulator and a randomly curved road path. Proc. of the 10th Int. Symp. on Advanced Vehicle Control, AVEC10.

Velenis, E., Katzourakis, D., Frazzoli, E., Tsiotras, P., \& Happee, R. (2010). Stabilization of Steady-State Drifting for a RWD Vehicle. Proc. of the 10th Int. Symp. on Advanced Vehicle Control, AVEC10, 820-825.

Velenis, E., Tsiotras, P. \& Lu, J. (2007a). Modeling aggressive maneuvers on loose surfaces: The cases of trail-braking and pendulum-turn. IEEE Proc. of the 2007 European Control Conference, Kos, Greece.

Velenis, E., Tsiotras, P. \& Lu, J. (2007b). Modeling aggressive maneuvers on loose surfaces: Data analysis and input parameterization. IEEE Proc. of the 2007 Mediterranean Conference on Control and Automation, Athens, Greece. 